\title{
Results from the Search-Lidar Demonstrator Project for Detection of Small Sea-Surface Targets
}

\author{
Johan C. van den Heuvel, Frank J.M. van Putten, Leo H. Cohen, Rob. A.W. Kemp, Gijs C. Franssen \\ TNO Defence, Security and Safety, Oude Waalsdorperweg 63, The Hague, The Netherlands
}

\begin{abstract}
Coastal surveillance and naval operations in the littoral both have to deal with the threat of small sea-surface targets. These targets have a low radar cross-section and a low velocity that makes them hard to detect by radar. Typical threats include jet skis, FIAC's, and speedboats. Previous lidar measurements at the coast of the Netherlands have shown a very good signal to clutter ratio with respect to buoys located up to $10 \mathrm{~km}$ from the shore where the lidar system was situated. The lidar clutter is much smaller than the radar clutter due to the smoothness of the sea surface for optical wavelengths, thus almost all laser light is scattered away from the receiver. These results show that due to the low clutter a search lidar is feasible that can detect small sea-surface targets. Based on these promising results a search-lidar demonstrator project has started end of year 2008. The system set-up of the search lidar demonstrator is presented and experimental results near the coast of Holland are presented. By using a high rep-rate laser the search time is limited in order to be useful in the operational context of coastal surveillance and naval surface surveillance. The realization of a search lidar based on a commercially available high power and high rep-rate laser is presented. This demonstrator is used to validate the system modeling, determine the critical issues, and demonstrate the feasibility.
\end{abstract}

Keywords: Laser, small targets, lidar, detection

\section{INTRODUCTION}

Detection of small sea-surface targets, like periscope tubes, jet skis, swimmers and small boats, is important both for naval and civil scenarios, e.g. 'man overboard', illegal immigration, drugs transport and asymmetric threats. However, the detection of these targets using current radar systems is difficult due to the small radar cross-section and low velocity of these targets and the presence of strong radar clutter from the sea surface.

A search lidar has unique properties for the detection of small surface targets due to the high resolution and the excellent signal to clutter ratio. In this way it is possible to detect small, relatively slow moving targets, while radar is limited by the low speed and the radar clutter of the water surface. The search lidar is complementary to modern surface radar (e.g. SeaStar) by detecting small and slow targets at short range, while the radar detects larger targets at longer ranges.

The principle of a search lidar consists of a scanning laser beam with a relatively small instantaneous field-of-view (IFOV) in combination with an optical receiver, aligned with the laser beam. Scanning is done in azimuth and elevation to cover the sea surface around the ship. The receiver measures part of the backward-scattered laser radiation from targets within the IFOV of the laser beam. Range information is obtained by using pulsed lasers in combination with a receiver that records the time-of-flight of the received laser pulse. A sketch of a basic search lidar is shown in Figure 1 with diagrams of the scanning system and the interaction at the sea surface.

Electro-Optical Remote Sensing, Photonic Technologies, and Applications III, edited by G. W. Kamerman, O. K. Steinvall, K. L. Lewis, R. C. Hollins, T. J. Merlet, G. J. Bishop, J. D. Gonglewski, Proc. of SPIE Vol. 7482, 748205 - (c) 2009 SPIE · CCC code: 0277-786X/09/\$18 - doi: 10.1117/12.830652 


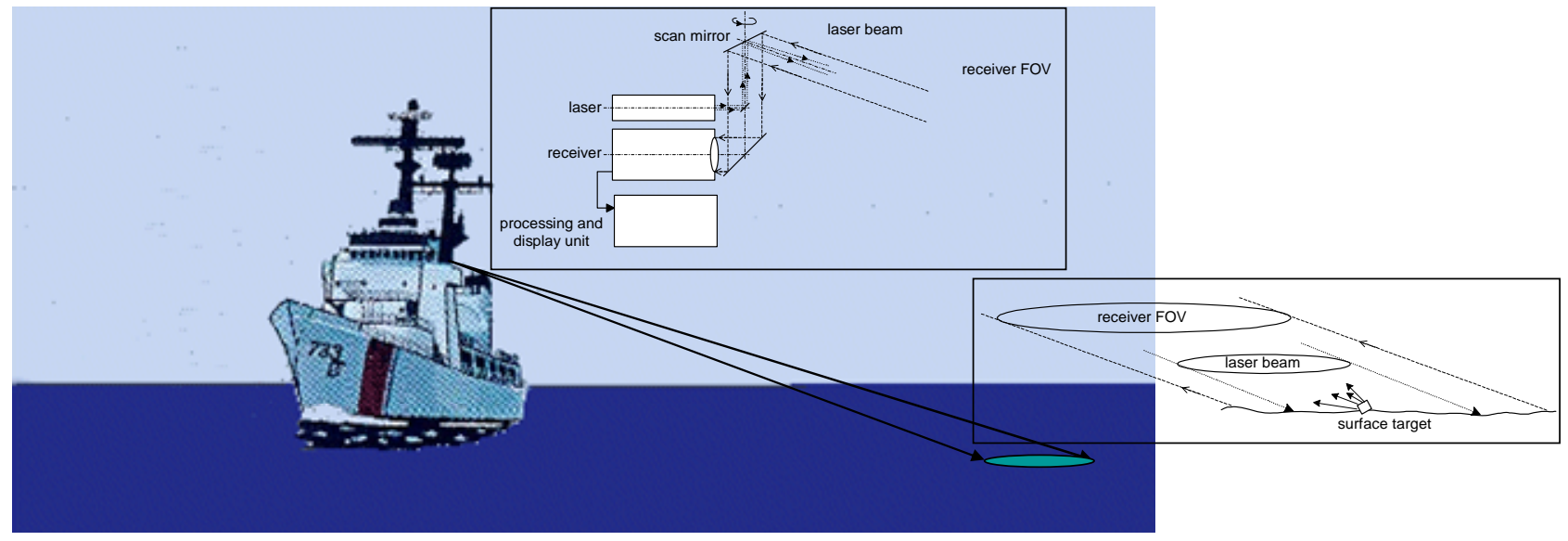

Figure 1: Sketch of a search lidar with diagrams of scanning system and sea-surface interaction.

In addition to the good detection potential for small targets, the search lidar has an excellent precision (resolution in both azimuth and range). This facilitates an unambiguous operational picture. Furthermore, this precision makes accurate weapon engagement possible.

In 2008 a National Technology Project called DELTA, started in the Netherlands. DELTA has the objective to demonstrate the potential of a search lidar for detection of small surface targets and to improve the technology for future implementation in coastal surveillance and on ships. This paper reports on the first results with a preliminary version of the demonstrator.

Future search-lidar systems can be installed at the coast for surveillance of coastal waters, harbours, and inland waterways. Additionally, the system can be placed on board a ship. At first, the system will be used in the military domain. Subsequently, the system will be installed in a civil environment where applications require an accurate situation awareness that includes small traffic.

\section{PREVIOUS LIDAR RESULTS}

\subsection{Results with a 1.06 micron atmospheric lidar}

To verify the search lidar concept, initial measurements were carried out on small buoys near Scheveningen harbor (North Sea coast, The Netherlands) at distances between 1 and $10 \mathrm{~km}$ with a $1.06 \mu \mathrm{m}$ system at a pulse energy of $200 \mathrm{~mJ}$ at $20 \mathrm{~Hz}$. Although the lidar operating at $1.06 \mu \mathrm{m}$ is an excellent tool for a feasibility study, it is not eye-safe. Therefore, the DELTA search lidar operates at a wavelength of $1.5 \mu \mathrm{m}$, which is absorbed in the eye and not focused on the retina.

A magnified portion of Hydrographic Map 1801.7 is shown on the left of Figure 2. The map (courtesy Royal Netherlands Navy, 'Dienst der Hydrografie') shows the buoys near the Scheveningen harbour entrance. The rectangles in the background of the map are approximately $1 \times 2 \mathrm{~km}^{2}$. The lidar was positioned at the end of the southern pier, near the green traffic light (near text 'Obtns'). Shown on the right side of Figure 2 is a view over the sea in NW direction from the southern harbour pier, with the lidar in the front and the buoys in the back. Red circles mark the four buoys of interest that are from left to right: Dr-B, Drain-W, Dr-A, and Drain-E. The ranges of the buoys are Dr-B at $1.4 \mathrm{~km}$, Drain-W at $1.9 \mathrm{~km}$, Dr-A at $1.2 \mathrm{~km}$, and Drain-E at $1.6 \mathrm{~km}$. 

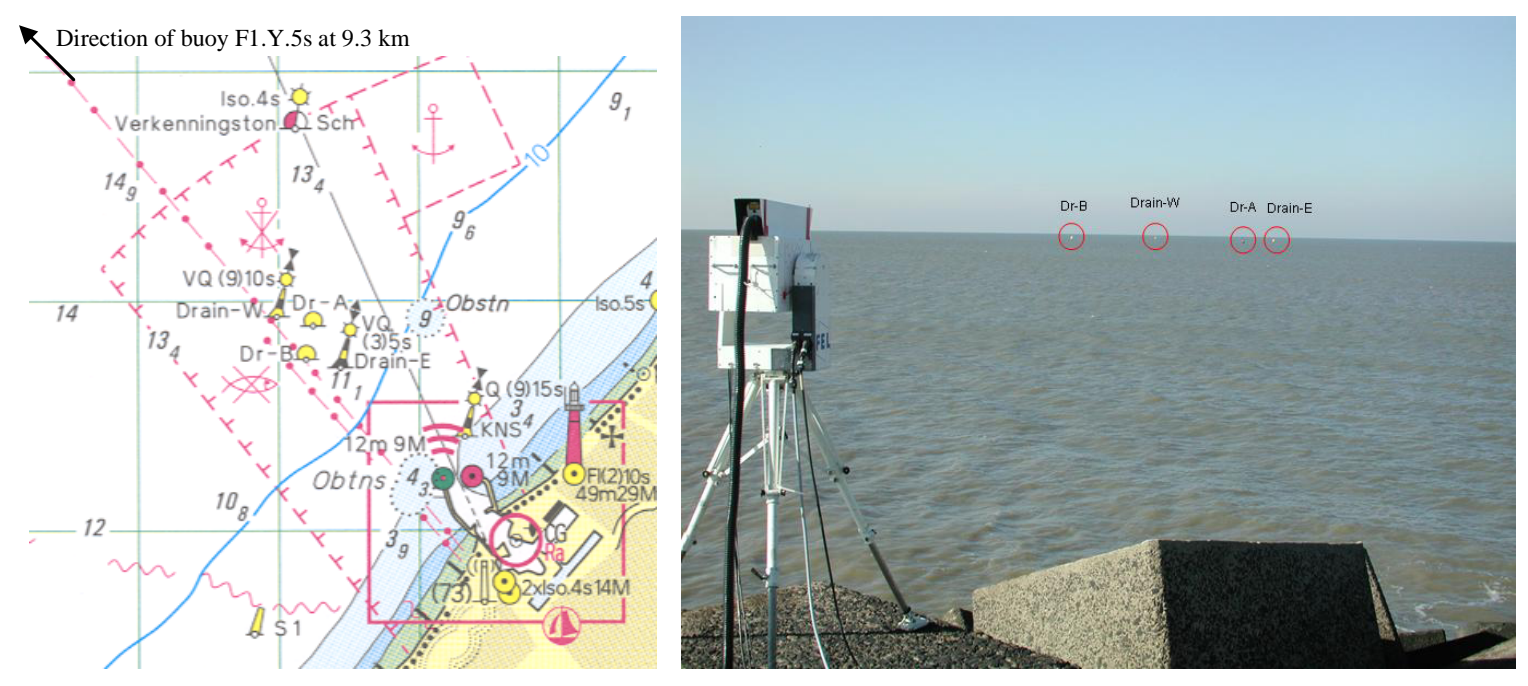

Figure 2: Magnified portion of Hydrographic Map 1801.7 (left) and the lidar situated at the end of the southern pier (right).

Horizontal lidar measurements were carried out at 10 different elevation angles from $+0.46^{\circ}$ degrees down to $-0.49^{\circ}$ to observe buoy reflections at different heights. Results obtained from one of these measurements under the elevation angle of $-0.24^{\circ}$ are presented in Figure 3. The lidar is in the right most position in this figure. Reflections from the buoys are indicated with red circles. Distances between the concentric range indicators are $500 \mathrm{~m}$. The regular fringes at ranges larger than $2 \mathrm{~km}$ (in the most left part in the figure) are reflections from the wave tops. The graph at the bottom is plotted with the same data but with another color scale showing only the strong reflection from the buoys.
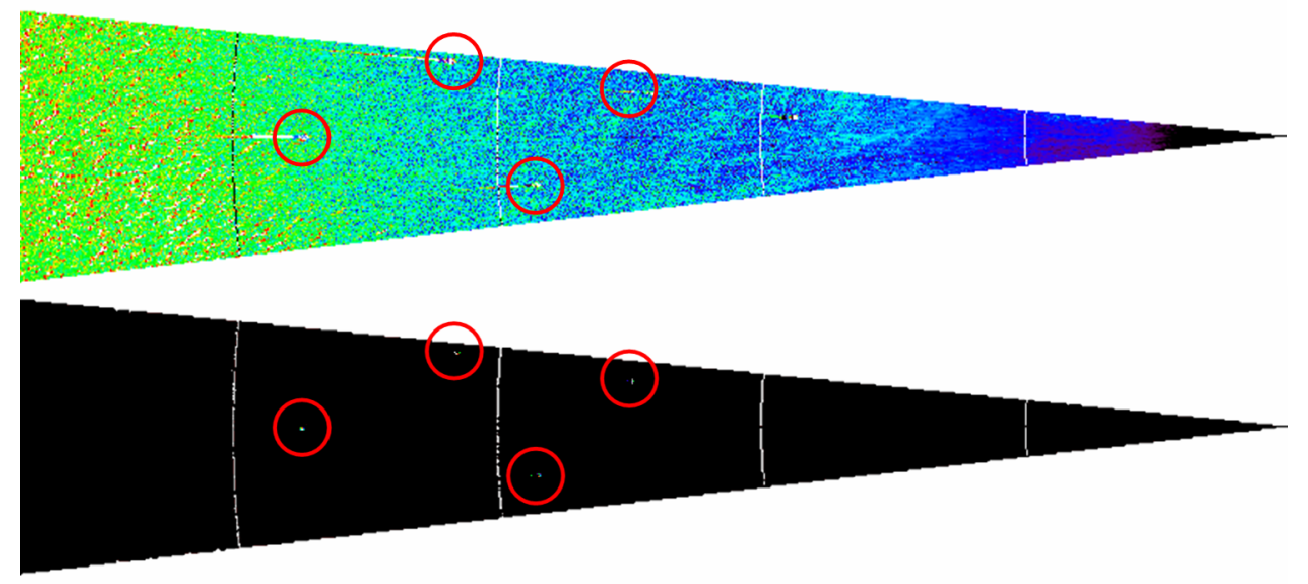

Figure 3: Results of a horizontal lidar scan over an azimuth angle interval of 12 degrees and at an elevation angle of $-0.24^{\circ}$. Two color scales are used. The top graph emphasizes the atmospheric backscatter while the bottom graph shows only the strong reflection from the four buoys.

During later experiments, the lidar could successfully measure the position and reflection of the buoy F1.Y.5s in northwesterly direction at a distance of $9.3 \mathrm{~km}$. The latter could not be observed with the naked eye under these prevailing conditions and only slightly using a binocular. Using the lidar, the buoy could be detected with a signal-tonoise ratio of about 140. A screen snapshot of the waveform recorder is shown in Figure 4. The lidar waveform presented in Figure 4 was recorded with a time base of $10 \mu \mathrm{s} / \mathrm{div}$ and a pretrigger of $10 \mu \mathrm{s}$. The gradual decrease in the signal between about $10 \mu \mathrm{s}$ and $60 \mu \mathrm{s}$ represents the atmospheric backscatter, attenuated by the geometric effect and the atmospheric transmission losses. Due to the application of an analog logarithmic amplifier, which suppresses large signal amplitudes and enhances low signal amplitudes, the large dynamic range of the signal could be covered within a single waveform. The relatively strong peak, visible at $62 \mu$ s after the pretrigger, comes from the buoy at $9.3 \mathrm{~km}$ from the lidar. 


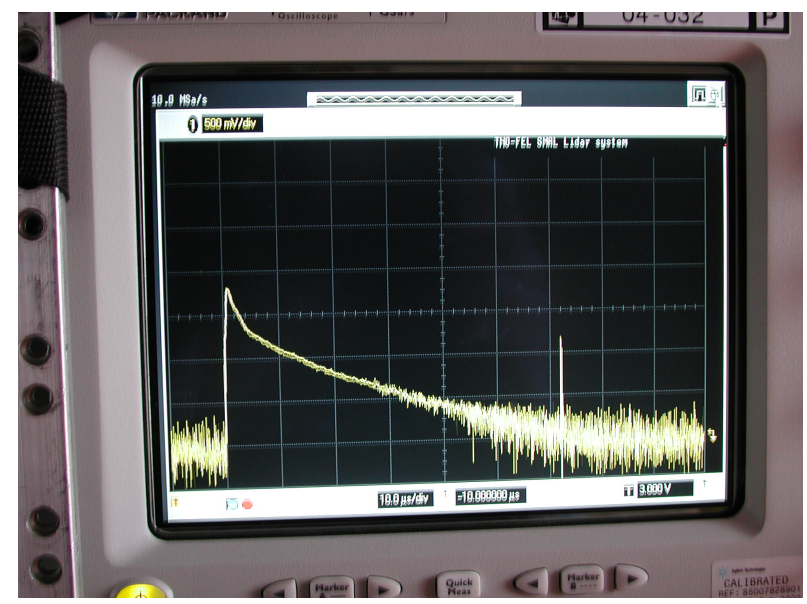

Figure 4: Photo of the oscilloscope screen with a lidar waveform showing the reflected signal from buoy Fl.Y.5s at $9.3 \mathrm{~km}$.

\subsection{Results with a 1.5 micron lidar}

The $1.5 \mu \mathrm{m}$ lidar was used at the same location as the $1.06 \mu \mathrm{m}$ to check whether the same favorable results could be achieved at this eye-safe wavelength. Fortunately, different references indicate that reflection charateristics of 1.06 and $1.5 \mu \mathrm{m}$ are often similar. ${ }^{1-4}$ The lidar system at $1.57 \mu \mathrm{m}$ is depicted in Figure 5. The green box contains the OPO laser wavelength converter, which converts the pump laser wavelength of $1.06 \mu \mathrm{m}$ into the more eye-safe wavelength of 1.57 $\mu \mathrm{m} .{ }^{5,6,7}$ The white box on top of the green box contains the receiver telescope and the detector. The white box right next to the green box is the $1.06 \mu \mathrm{m}$ pump laser. The lidar system was operated with maximum pump pulse energy of $205 \mathrm{~mJ}$ at a rep-rate of $10 \mathrm{~Hz}$. The output from the transmitter at $1570 \mathrm{~nm}$ was around $30 \mathrm{~mJ}$. Since this system does not have a logarithmic receiver for the atmospheric return signal, we can only show the linear plot of the return of the buoys. Figure 6 shows the lidar return signals for two buoys, which clearly show a good signal-to-noise ratio.

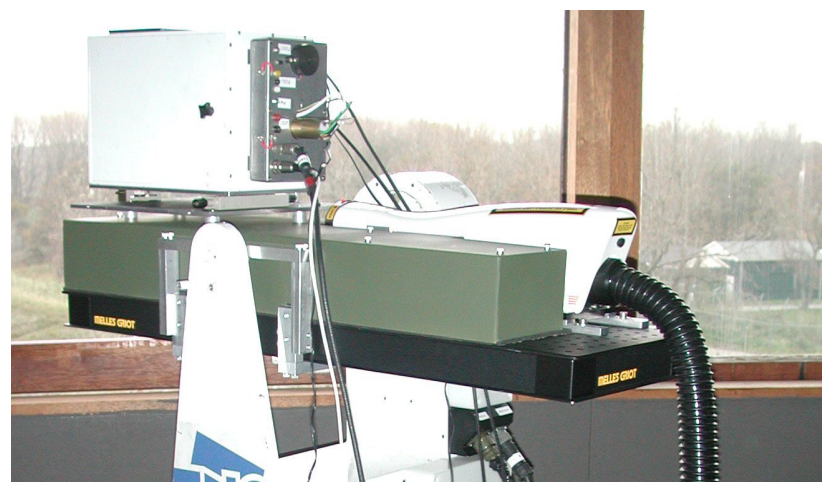

Figure 5: 1.5 micron lidar system 


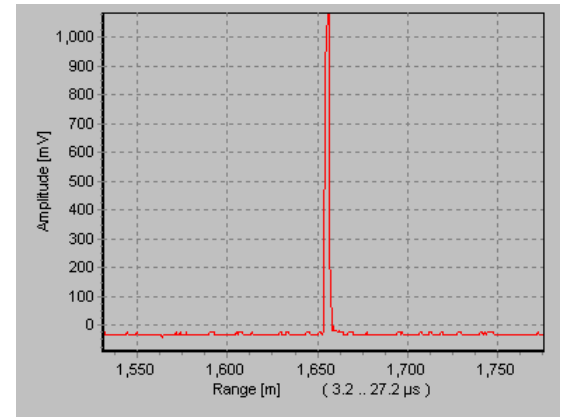

Drain-E

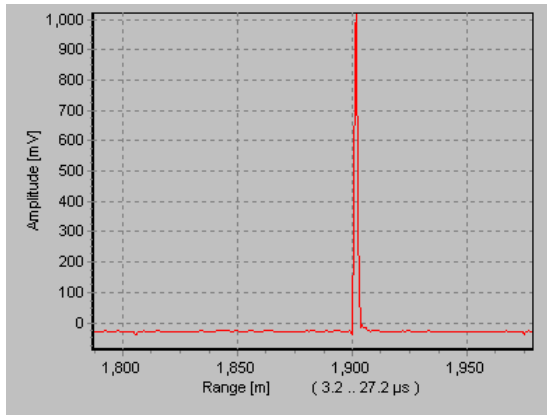

Drain-W

Figure 6: Laser return signals of two buoys measured with the $1.5 \mu \mathrm{m}$ lidar.

Since the lidar is eye-safe, we can also aim the system at manned platforms without risk of eye damage. Figure 7 shows that is it feasible to detect a small sailing boat at $3.4 \mathrm{~km}$. Given the good signal-to-noise ratio, much larger ranges are possible. Ranges up to $10 \mathrm{~km}$ were achieved with this lidar at a NATO trial in Norway in the summer of $2007 .^{8}$
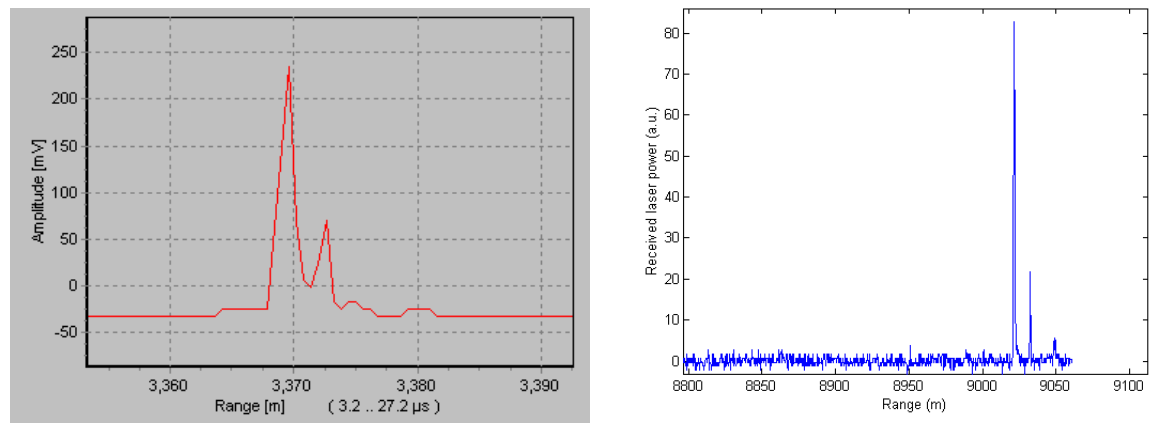

Figure 7: Laser range profile of a small sailing boat at $3.4 \mathrm{~km}$ (left) and a large ship at $9 \mathrm{~km}$ (right).

\section{DEFINITION SEARCH-LIDAR DEMONSTRATOR}

\subsection{System block-diagram}

An overview of the search-lidar system components is presented in Figure 8. Essential lidar system components are the transmitter and receiver elements. The transmitter is a laser which emits short light pulses (of the order of nanoseconds) with a high repetition rate (of the order of kilohertz). The short pulses allow for a good distance resolution, while the high repetition rate is essential for fast scanning of the region of interest. The receiver part of the setup consists of a fast photodetector (of the order of tens or hundreds of megahertz) in combination with suitable focussing optics. Laser beam divergence and receiver field-of-view (FOV) are well-defined and partly or fully overlap on the target. 


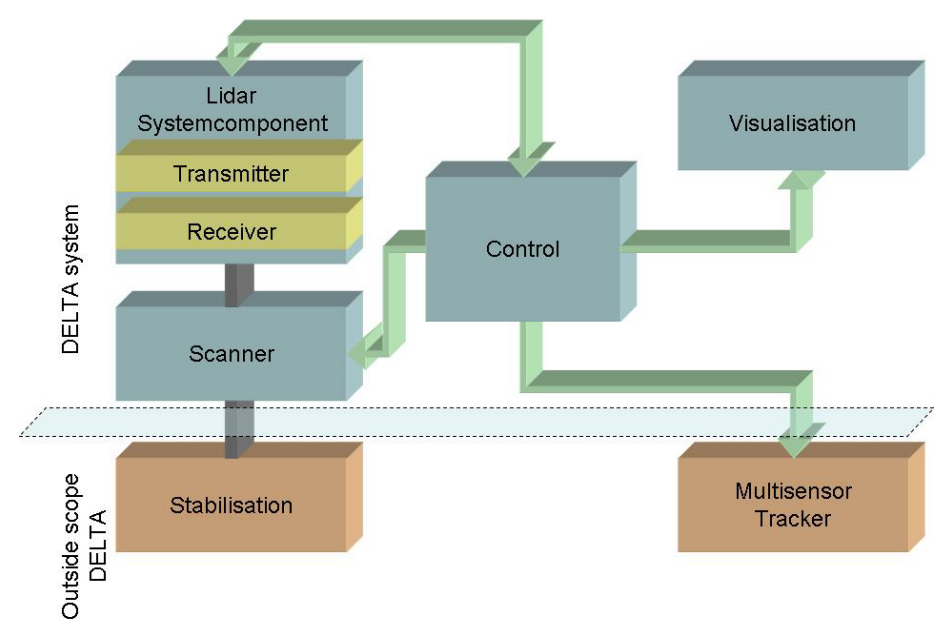

Figure 8: Overview of search-lidar system components.

A search-lidar is constructed by placing the lidar system is on a scanning platform. Parameter values related to the lidar system components and scanning system will be discussed in more detail in the following sections, where we will be concerned with the system requirements. As indicated in Figure 8, aspects of search-lidar which are related to multisensor tracking and stabilisation of the signal, for example in the case of a search-lidar system placed on board of a ship, are outside of the scope of the DELTA project.

\subsection{Search time}

A crucial requirement is that the search time is short enough to detect sea-surface targets in time. Since the search space is limited (namely at small elevation angles below the horizon) and surface targets move relatively slow, this required search time is long enough for a search lidar to be feasible. Note that the search lidar is meant for slow moving surface targets that are difficult to detect for radar.

The search time is proportional to the number of laser pulses required for monitoring the sea surface around the search lidar position. For a horizontal scan (or azimuth scan) over angle $\theta$, the number of pulses is

$$
N_{A}=\frac{\theta}{\psi},
$$

where $\psi$ is the laser beam divergence (and identical to the instantaneous field-of-view). For a full azimuth scan of $2 \pi$ radians and a beam divergence of $1 \mathrm{mrad}$, we find that at least 6300 laser pulses are required. If the search lidar is mounted at a height $h$, then the geometrical horizon is a function of the radius of the earth $R_{E}$ :

$$
R_{G H}=\sqrt{2 R_{E} h} .
$$

For our low power system, a maximum range of $2 \mathrm{~km}$ is feasible. The corresponding minimum mounting height is 0.31 meter using equation (2) and the $R_{E}$ of $6400 \mathrm{~km}$. For a search lidar with a maximum range of around $10 \mathrm{~km}$, the minimum mounting height for full range is $10 \mathrm{~m}$. The elevation scan from minimum range $R_{\min }$ to the horizon needs the following number of laser pulses:

$$
N_{E}=\frac{h}{R_{\min } \psi} .
$$

A minimum range of $150 \mathrm{~m}$ means a depression angle of 2 mrad leading to two scans in the elevation direction based on the mounting height of 0.3 meter. Multiplying the number of horizontal and the vertical scans, we find a total number of 12.600 laser pulses.

The search time based on 12.600 laser pulses is around six seconds for our current low power $2 \mathrm{kHz}$ laser. If we take into account that coastal surveillance will require a semi-circle scan, a search time of three seconds is obtained. This search time is sufficiently low to be applicable in coastal surveillance. 
If the system is mounted at a height of $10 \mathrm{~m}$ above the sea surface, the geometric horizon is positioned at about $11.3 \mathrm{~km}$ and a depression angle of about $2 \mathrm{mrad}$. A minimum range of $150 \mathrm{~m}$ means a depression angle of $67 \mathrm{mrad}$ leading to about 65 pulses with a 1 mrad laser beam for the vertical scan. Thus, a full coverage between $150 \mathrm{~m}$ and $11.3 \mathrm{~km}$ requires at least $6280 \times 65=408,000$ pulses. Using a $10 \mathrm{kHz}$ repetition rate laser the cycle time is about $41 \mathrm{~s}$. A shorter cycle time can be obtained by using a scanner with a lidar FOV that increases with decreasing range. Another option is to increase the laser beam divergence in order to reduce the number of shots that is required for a full surface scan. A beam divergence of $2 \mathrm{mrad}$ would lead to a cycle time of about $10 \mathrm{sec}$. This seems acceptable for a scenario with relatively slow moving targets.

It is clear from these two cases that the search time increases rapidly with increased mounting height. Therefore, the mounting height should be as low as possible in the given application. However, the mounting height can not be lower than dictated by the required geometric horizon. Furthermore, the height must be high enough to overcome tidal variations (or use an adjusting height) and wave heights.

\subsection{Detection ranges}

The range performance of search lidars can be modeled by taking into account the system parameters, the target properties and the transmission of the atmosphere. These parameters can be used in relatively straightforward laser range finder equations (see, e.g., Ref. [8]). Two different situations must be distinguished. The first one is based on the assumptions that the cross-section of the illuminating laser beam at the position of the target is small compared to the dimensions of the target, whereas the second equation must be used when the cross-section of the laser beam is larger than the cross-section of the target. These models are presented in Equations (4) and (5) respectively:

$$
\begin{array}{ll}
P_{D}=\frac{\eta}{R^{2}} \cdot \frac{P_{L} A_{D} T_{0}}{\pi} \cdot T_{A}^{2} & \text { for 'large' targets and, } \\
P_{D}=\frac{\eta A_{T}}{R^{4}} \frac{4 P_{L} A_{D} T_{O}}{\pi^{2} \Delta \psi^{2}} T_{A}^{2} & \text { for 'small' targets. }
\end{array}
$$

Here the parameter are

$\begin{array}{lll}A_{D} & - & \text { receiver area }\left[\mathrm{m}^{2}\right] ; \\ A_{T} & - & \text { target area in }\left[\mathrm{m}^{2}\right] ; \\ P_{D} & - & \text { received signal }[\mathrm{W}] ; \\ P_{L} & - & \text { emitted laser power }[\mathrm{W}] ; \\ R & - & \text { range finder }- \text { target distance }[\mathrm{m}] ; \\ T_{O} & - & \text { transmission of the optics }[0 . .1] ; \\ T_{A} & - & \text { one-way atmospheric transmission }[0 . .1] \\ \eta & - & \text { target equivalent reflection coefficient }[-] ; \\ \Delta \psi & - & \text { laser beam divergence }[\mathrm{rad}] .\end{array}$

Targets may be identified when the returned signal is stronger than a selected minimum level or threshold, which is a function of an acceptable average false alarm rate (FAR) and the receiver noise level. In turn, the probability of detection (PD) can be calculated from the strength of the returned target signal, the noise level and the selected threshold, see, e.g., Ref. [10]. Because the receiver noise characteristics depend on the background radiation, optimum threshold settings should be carried out adaptively. Atmospheric transmission can be estimated from the visibility and by applying the proposed wavelength correction, e.g., following Ref. [11]:

$$
\eta_{\text {atm }}=\exp (-\mu R) \text { with } \mu=\frac{3.912}{V}\left(\frac{\lambda_{r}}{\lambda}\right)^{q},
$$

where $\mu$ is the atmospheric extinction coefficient, $V$ is the visibility, and $\lambda$ is the laser wavelength. The constant $\lambda_{r}$ is $0.55 \mu \mathrm{m}$ and $q$ is given by:

$$
q=0.585 \mathrm{~V}^{1 / 3} .
$$

Finally, it is important to identify the optical power threshold value $P_{t h r}$ that can be detected by the receiver, which depends on the noise-equivalent power (designated NEP) of the detector: 


$$
P_{t h r}=N E P \sqrt{B W} S N R \text {. }
$$

In this equation, $B W$ signifies the detector bandwidth and $S N R$ is the signal-to-noise ratio necessary for reliable detection.

By using Eq. (8) and detector parameters, we can calculate the optical power threshold appropriate for a given scenario. Eqs. (4) through (7) can then be used to find, for given laser parameters, the detection range at which this threshold can be exceeded.

We will consider four different laser modules. Of these modules, two are to be used in the DELTA search-lidar experiments (lasers 1 and 3), while two others are of potential interest (laser 2 and 4). Emission wavelengths of the lasers are in all cases about $1.5 \mu \mathrm{m}$. The main difference between these lasers is the pulse energy, as is indicated in Table 1. Repetition rates are high enough to allow for the design of a time-efficient search-lidar, whereas the short pulse durations gives an excellent distance resolution.

Table 1: Four laser systems relevant for the search-lidar transmitter component.

\begin{tabular}{|l|c|c|c|}
\hline & Pulse energy & Repetition rate & Pulse duration \\
\hline 1. Cobalt laser & $7 \mu \mathrm{J}$ & $2 \mathrm{kHz}$ & $3 \mathrm{~ns}$ \\
\hline 2. Fiber laser & $200 \mu \mathrm{J}$ & $5 \mathrm{kHz}$ & $10 \mathrm{~ns}$ \\
\hline 3. YAG-pumped OPO I & $1 \mathrm{~mJ}$ & $2 \mathrm{kHz}$ & $10 \mathrm{~ns}$ \\
\hline 4. YAG-pumped OPO II & $10 \mathrm{~mJ}$ & $10 \mathrm{kHz}$ & $10 \mathrm{~ns}$ \\
\hline
\end{tabular}

In order to estimate the detection ranges of these laser systems, detector parameters have to be known. For evaluation of the laser systems, we assume a detector $N E P$ of $2.5 \cdot 10^{-13} \mathrm{~W} / \sqrt{\mathrm{Hz}}$, a $B W$ of $200 \mathrm{MHz}$, and a $S N R$ of 7 . The receiver diameter is $10 \mathrm{~cm}$; the transmission of the optics and target equivalent reflection coefficient are 0.5 and 0.05 , respectively. Estimated detection ranges are displayed for assumed visibilities of $30 \mathrm{~km}$ (Table 2) and $1 \mathrm{~km}$ (Table 3), and for two different objects: a fishing boat with a side view area of $80 \mathrm{~m}^{2}$ and a speed boat with a side view area of $5 \mathrm{~m}^{2}$.

Table 2: Estimated detection ranges for two different objects and for different laser systems with an assumed visibility of $30 \mathrm{~km}$.

\begin{tabular}{|c|c|c|c|c|}
\hline & $\begin{array}{c}1 . \text { Cobalt } \\
(7 \mu \mathrm{J})\end{array}$ & $\begin{array}{c}2 . \text { Fiber } \\
(200 \mu \mathrm{J})\end{array}$ & $\begin{array}{c}3 . \text { OPO I } \\
(2 \mathrm{~mJ})\end{array}$ & $\begin{array}{c}4 . \text { OPO II } \\
(10 \mathrm{~mJ})\end{array}$ \\
\hline $\begin{array}{c}\text { Fishing boat } \\
\left(80 \mathrm{~m}^{2}\right)\end{array}$ & $1.3 \mathrm{~km}$ & $6.2 \mathrm{~km}$ & $13.1 \mathrm{~km}$ & $18.5 \mathrm{~km}$ \\
\hline $\begin{array}{c}\text { Speed boat } \\
\left(5 \mathrm{~m}^{2}\right)\end{array}$ & $1.3 \mathrm{~km}$ & $4.1 \mathrm{~km}$ & $7.0 \mathrm{~km}$ & $10.1 \mathrm{~km}$ \\
\hline
\end{tabular}

Table 3: Estimated detection ranges for two different objects and for different laser systems with an assumed visibility of $1 \mathrm{~km}$.

\begin{tabular}{|c|c|c|c|c|}
\hline & $\begin{array}{c}1 . \text { Cobalt } \\
(7 \mu \mathrm{J})\end{array}$ & $\begin{array}{c}2 . \text { Fiber } \\
(200 \mu \mathrm{J})\end{array}$ & $\begin{array}{c}3 . \text { OPO I } \\
(2 \mathrm{~mJ})\end{array}$ & $\begin{array}{c}4 . \text { OPO II } \\
(10 \mathrm{~mJ})\end{array}$ \\
\hline $\begin{array}{c}\text { Fishing boat } \\
\left(80 \mathrm{~m}^{2}\right)\end{array}$ & $0.5 \mathrm{~km}$ & $0.9 \mathrm{~km}$ & $1.3 \mathrm{~km}$ & $1.6 \mathrm{~km}$ \\
\hline $\begin{array}{c}\text { Speed boat } \\
\left(5 \mathrm{~m}^{2}\right)\end{array}$ & $0.5 \mathrm{~km}$ & $0.9 \mathrm{~km}$ & $1.3 \mathrm{~km}$ & $1.6 \mathrm{~km}$ \\
\hline
\end{tabular}

It can be observed from the numbers in Table 2 and Table 3 that the detection range of the lasers differs considerably, especially for atmospheric conditions in which the visibility is good. Lasers with $\mathrm{mJ}$-order pulse energies can then easily exceed detection ranges of $10 \mathrm{~km}$. These ranges are close to or even larger than the geometrical horizon, which is about $11 \mathrm{~km}$ for a lidar positioned $10 \mathrm{~m}$ above the sea-level.

The use of low-pulse-energy lasers has the advantage that such systems are often quite compact and therefore easier to handle. Also, they tend to be more robust. As can be seen from the data in Tables 2 and 3, $\mu \mathrm{J}$-order pulses can still reach detection ranges exceeding a kilometer, which makes these lasers suitable for applications in scenarios taking place in settings like a harbour. 
As mentioned above, laser systems 1 and 3 are to be used for experiments performed in the framework of the DELTA search-lidar project. Laser system 1 has the advantage of being small and straightforward to use. It is used in the DELTA project for lidar observations at relatively short distances (up to around $1 \mathrm{~km}$ ) and presents a good possibility for shortrange search-lidar. System 3 has a pulse energy which is three orders of magnitude higher. It can therefore observe targets at much larger distances. In future experiments performed in the framework of DELTA, long-range search-lidar measurements will use system 3 .

Laser systems 2 and 4 are discussed here because of their potential for future search-lidar systems. The fiber laser (system 2) presents a promising option for future applications, especially because this type of laser is relatively small and robust. Pulse energies that can be obtained at about $1.5 \mu \mathrm{m}$ and $\mathrm{kHz}$ frequencies are not as high as in OPO-based systems, but significantly higher than in a cobalt laser. Laser system 4 is included to show the parameters of a realistic but more expensive laser system for higher range and shorter search time. Indeed, a pulse energy of $10 \mathrm{~mJ} \mathrm{at} 10 \mathrm{kHz}$ implies an average power of $100 \mathrm{~W}$, which requires more sophisticated technology.

\section{FIRST DEMONSTRATOR RESULTS}

In March 2009 a number of search-lidar experiments were performed with a preliminary version of the DELTA demonstrator again at the Scheveningen harbor site. Figure 9 shows a photograph of the four buoys from the position of the search lidar; the right side of the figure shows the ladar screen with three detections at the angle of $180 \mathrm{deg}$. We will use the term ladar screen since it has a close similarity with a radar screen. Another term could be lidar screen, however, the term lidar plot or screen is often used for showing atmospheric backscatter from aerosols and is therefore avoided. The fourth buoy at $1.9 \mathrm{~km}$ is too small and too far away for the very low-power of the Cobalt laser that was used.
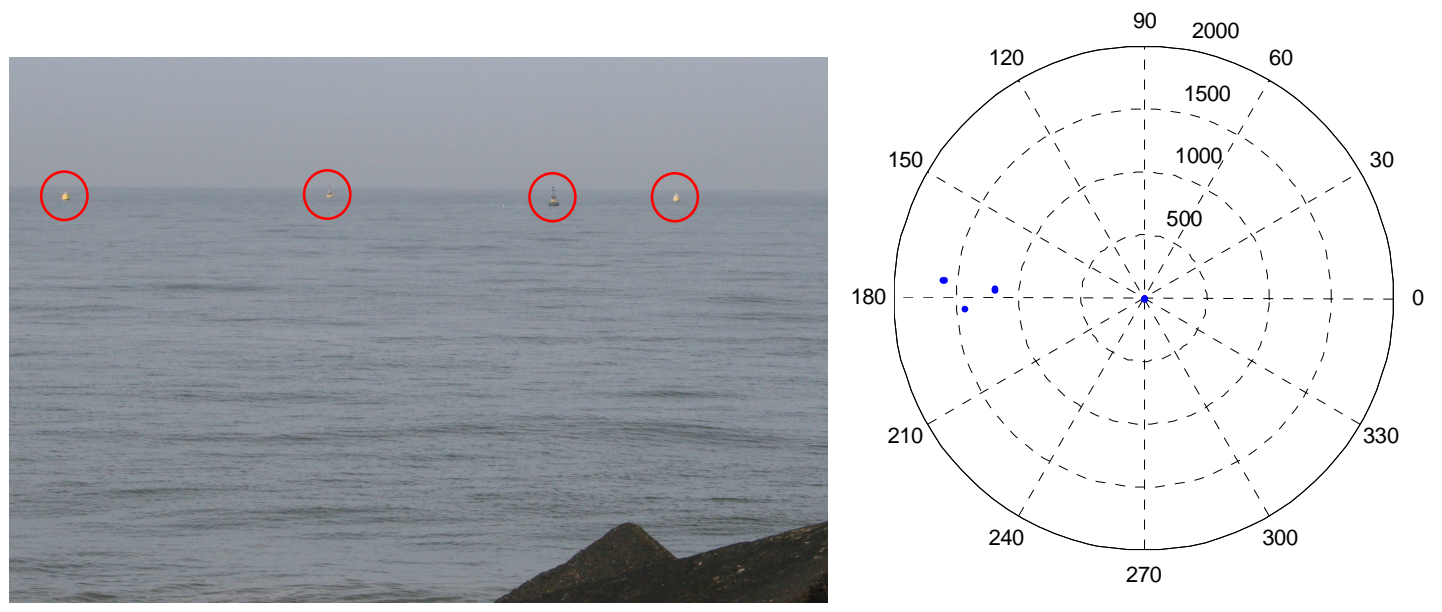

Figure 9: Photograph and ladar screen of the four buoys (three visible with lidar).

Due to the very low power of the laser, the received laser power was also very low. In order to detect the buoys we had to put the detection threshold very low and only slightly above the detector noise. This resulted in some false alarms. However, false alarms could be easily eliminated since the real targets gave two or more detections for each laser scan due to the small beam diameter resulting in several consecutive laser pulses on target.

Figure 10 shows a photograph and ladar plot of a small fishing boat at $1.2 \mathrm{~km}$ at angle $180 \mathrm{deg}$. The boat is quite small, but it is still detectable beyond one kilometre. 

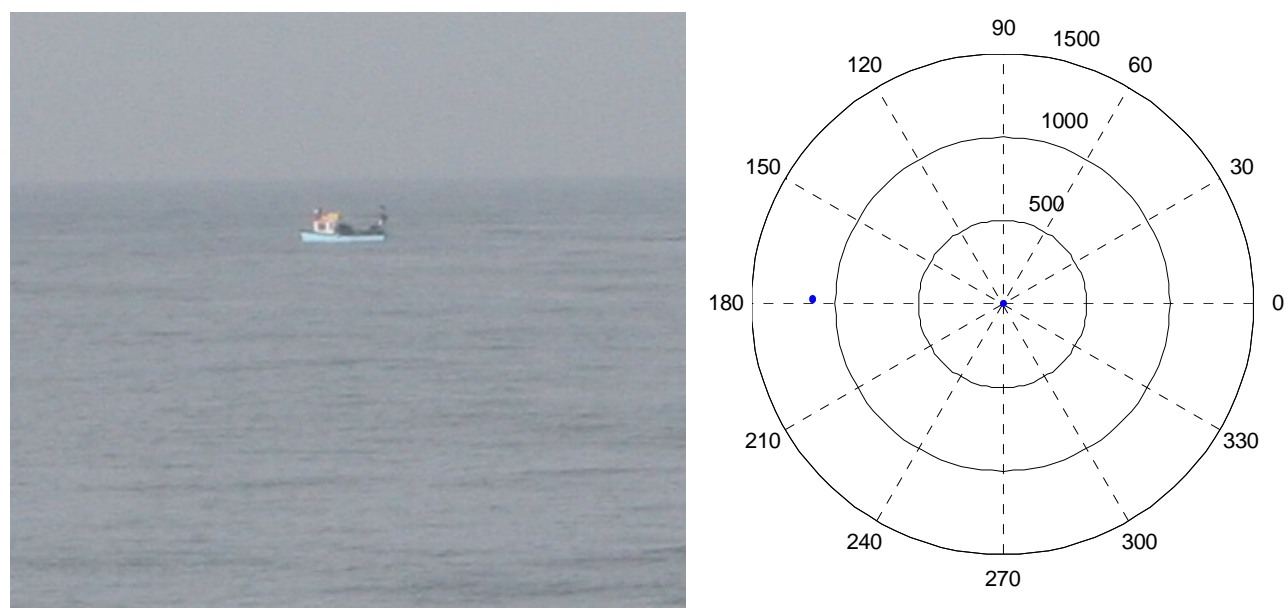

Figure 10: Photograph and ladar screen of a small fishing boat at $1.2 \mathrm{~km}$.

Figure 11 and Figure 12 show the photograph and the detections from the north side of Scheveningen harbour. Note that we scanned a larger angle than in the previous measurement of the section above. In addition, the coast line is visible on the ladar screen. Buildings near the coast beyond $1.5 \mathrm{~km}$ can be detected.

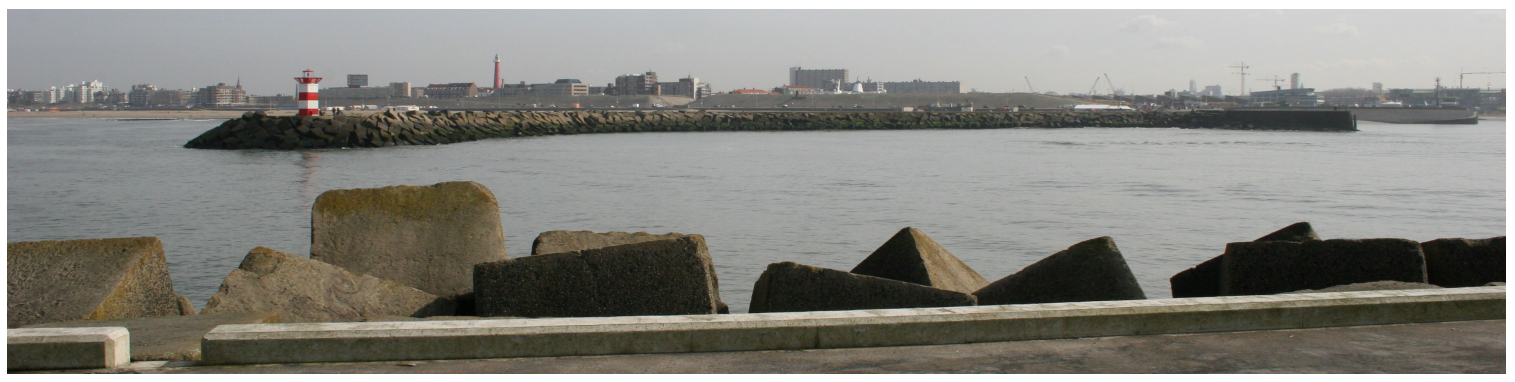

Figure 11: North side of Scheveningen harbour.

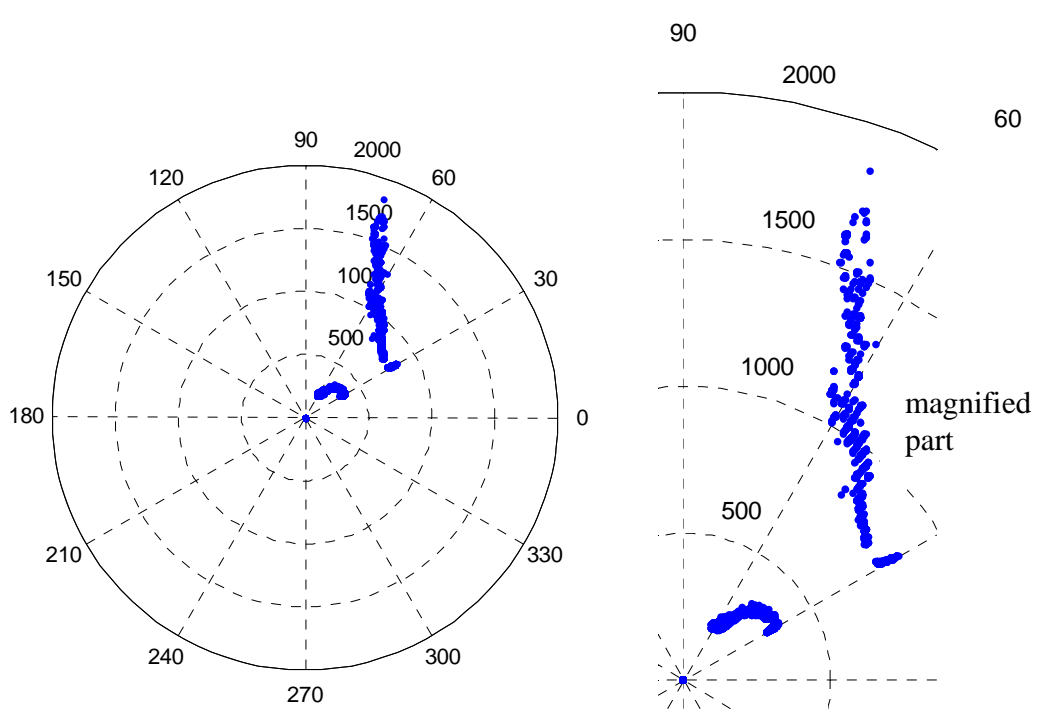

Figure 12: Ladar screens of the North side of Scheveningen harbour (detections of the left are from the coast line north of the harbour).

A large fishing ship is shown in Figure 13. The ladar screen shows a track of the ship due to the multiple scans that take some time and as a result the detections form a line. 

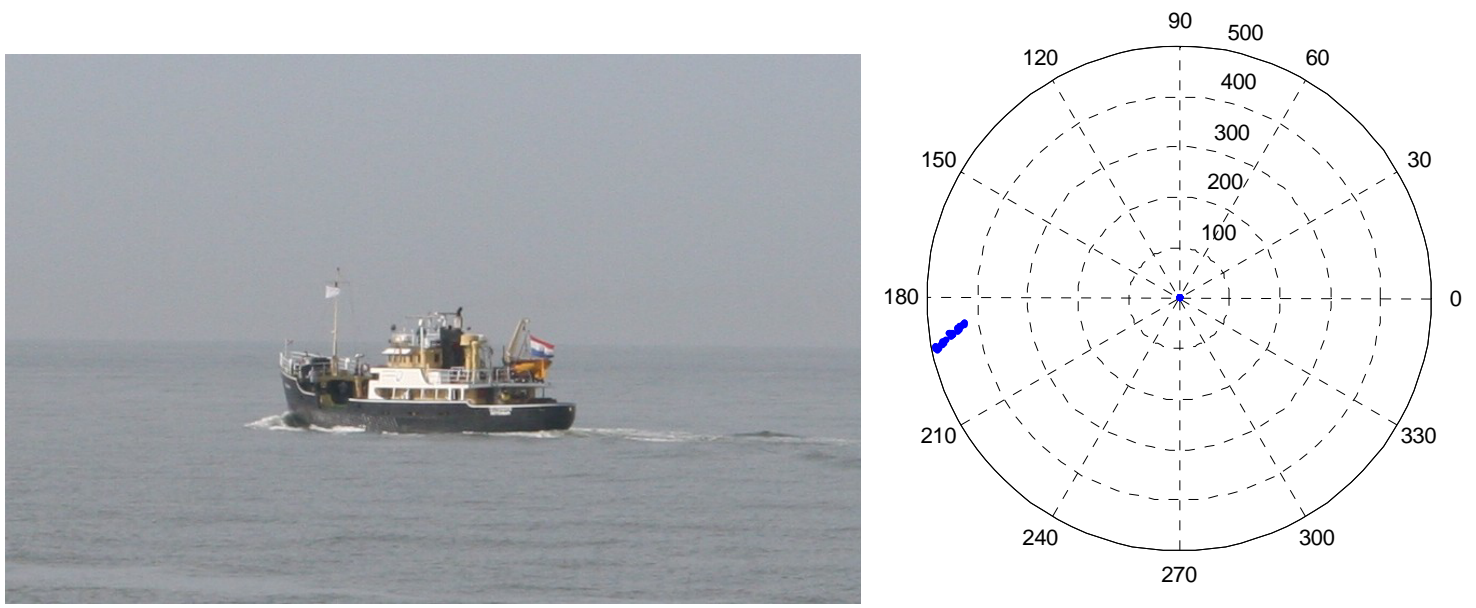

Figure 13: Photograph and ladar screen of a large fishing ship at $500 \mathrm{~m}$ (note that a track is formed from multiple detections).

A critical issue for a search-lidar is the required search time. The system uses the $1600 \mathrm{~Hz}$ repetition rate of the Cobalt laser. The scanning platform has a considerable speed of $50 \mathrm{deg} / \mathrm{s}$, however, some time is required to reach this speed. It order to test the search time, the search lidar scanned a quadrant of 90 degrees using 6 elevation steps. The search time was 22 seconds. An easy calculation shows that the average speed of the platform is $25 \mathrm{deg} / \mathrm{s}$ (90 times 6 divided by 22). Clearly, the scanner is not operating at maximum speed. This is due to the time required for decelerating and accelerating.

Figure 14 shows a target: the small fishing boat encountered before and two ladar screen at two different times. In these ladar screens the two extreme azimuth angles of the laser beam are indicated by red lines. It is clear that the small fishing boat is detected.
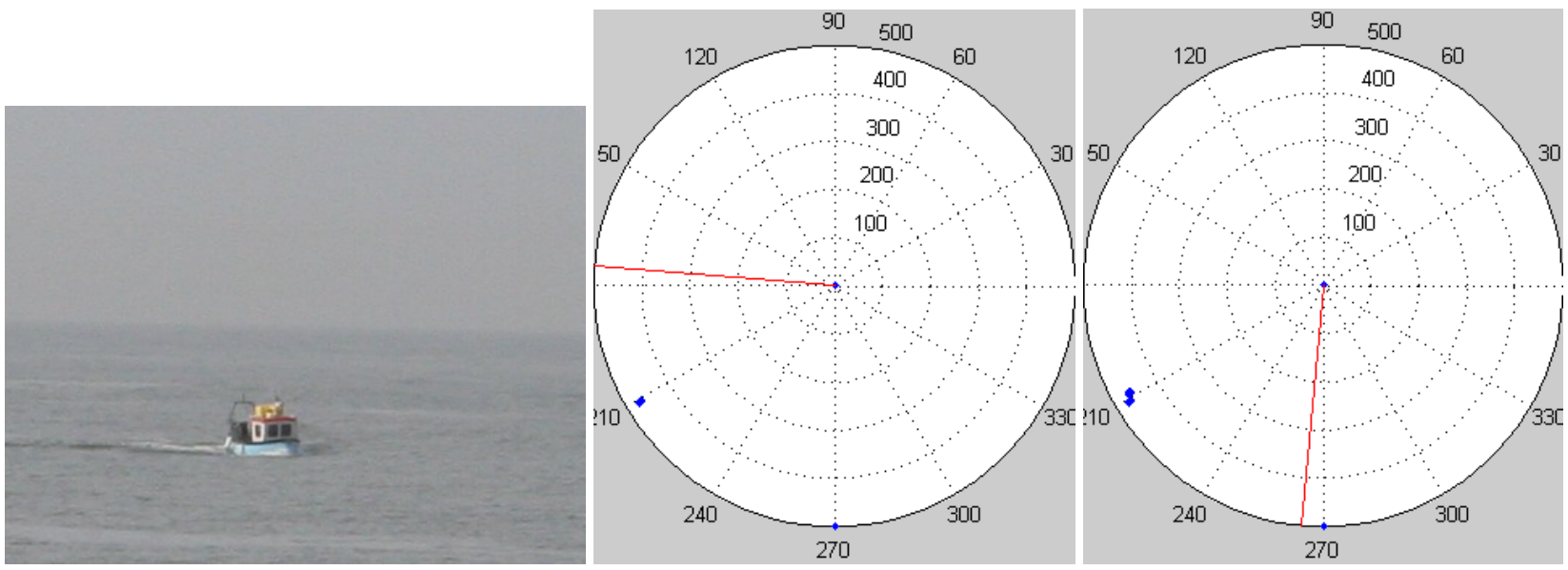

Figure 14: Small fishing boat at $500 \mathrm{~m}$ and two ladar screens showing the laser line as a red line. Left and right screens are the extremes of the scanning showing a quadrant scan (scan angle between 175 and 265 degrees)..

In order to shorten the search time in further tests and demonstrations, we will investigate the possibility of using fewer elevation scans. Note that using two elevation scans instead of six will reduce the search time to about 7 seconds. Furthermore, this search time will not increase substantially if we go to larger scan angles since the scanner will then operate closer to maximum speed. 
For a production version of a search lidar, it is most likely that a scanning mirror will be used instead of a scanning platform. This will remove the restriction in maximum speed and, in particular, acceleration. However, mounting a scan mirror is beyond the scope of the DELTA project.

\section{CONCLUSIONS}

Previous experiments with two systems, the $1.06 \mu \mathrm{m}$ lidar and the $1.5 \mu \mathrm{m}$ laser range profiler, have shown that the reflection of laser pulses from the sea surface is very low. This forms the basis for the concept of a search-lidar for the detection of surface targets. Both systems have shown good detection properties up to $10 \mathrm{~km}$ range.

The results of a search-lidar demonstrator have been presented. The laser system that detects small targets at the sea surface at a range up to $2 \mathrm{~km}$ at good visibility is feasible with current laser technology. Such a system will have a search time of a few seconds. Longer ranges are also possible for instance up to $10 \mathrm{~km}$. For these ranges, a higher power laser is required which is currently commercially available.

\section{ACKNOWLEDGEMENTS}

We thank Leo Böhmer of HITT Traffic and Chera Bekker of Thales Netherlands for discussions on the application in coastal surveillance and naval operations. We further thank Lt Menno Smeelen of the Netherlands Ministry of Defence for supporting this work in the NTP project DELTA.

\section{REFERENCES}

1. Suits, G.H., "Natural sources", Chapter 3 in W.L.Wolfe and G.J. Zissis, Eds., The infrared handbook (1978).

2. Lillesand, T.M., and Kiefer, R.P., [Remote sensing and image interpretation], New York: John Wiley \& Sons, (1973).

3. Rowan, L.C., Goetz, A.F.H., and Ashley, R.P., "Discrimination of hydro thermally altered and unaltered rocks in visible and near infrared multispectral images," Geophysics 42(3), 522-535, 1977.

4. Kahle, A.B., [Measuring spectra of arid lands, in Deserts and arid lands], El-Baz. F, Eds., pp. 195-217, Martinus Nijhoff Publishers, The Hague, The Netherlands, (1984).

5. Burnham, R.L., Kasinski, J.L., Marshall, L.R., "Eye-safe laser systems”, US Patent No. 5181211, (1993).

6. G.A. Rines, D.G. Rines, P.F. Moulton, "Efficient, High-Energy, KTP Optical Parametric Oscillators Pumped with 1 $\mu \mathrm{m}$ Nd-Lasers", OSA Proceedings on Advanced Solid State Lasers, Washington DC, Vol. 20, pp. 461-463, (1994).

7. B. Boulanger, M.M. Fejer, "Study of gray-tracking at 1064, 532, and 355 nm", Applied Physics Letters 65 (19) (1994).

8. Heuvel, J.C. van den, et al. "Experimental Validation of Ship Identification with a Laser Range Profiler", Proc. NATO MSS, March 2008 in Orlando, Florida USA.

9. A.V. Jelalian, 'Laser Radar Systems', Artech House, 1992.

10. R.W. Byren, 'Laser Rangefinders', in The Infrared \& Electro-Optical Systems Handbook Volume 6, Chapter 2, pp. 77-116, 2nd edition, 1996.

11. F. Löhle, "Über die Lichtstreuung im Nebel," Physik. Zeitschr. XLV, p. 199-204, 1944. 